\title{
CLINICAL TESTS FOR BINOCULAR VISION
}

\author{
JOHN LEE and ANN McINTYRE \\ London
}

As is well known, Claud Worth originally suggested that there are three grades of binocular function. His most basic grade was simultaneous macular perception, this being followed by fusion; finally, the highest grade of binocular function was claimed to be that of stereopsis. This 'wedding cake' model of binocular function is probably somewhat misleading and difficult to relate to the information gained from the tests of binocular function that are normally used in orthoptic examination and in screening. The intention of this paper is to discuss practical clinical points with regard to the detection and assessment of binocular function, in particular in younger and preverbal patients. It represents the practical experience and views of the authors and does not attempt to be a comprehensive review of all methods that have ever been described.

\section{THE NON-VERBAL YOUNG CHILD}

Much may be gained from simple external examination of young children. They are usually quite happy to look at an interesting fixation light, or indeed at their mother's face while a fixation light is held at an appropriate distance from their eyes, and it is usually possible to state whether the corneal reflexes appear to be symmetrical. The majority of cases of strabismus in small children tend to have fairly large angles, so the demonstration of symmetrical corneal reflexes is already a substantial step towards the assumption of probable binocular function.

An abnormal head posture is in general a sign that binocularity is being maintained in the presence of an incomitant deviation. It is typical that the head is placed in the position where the eyes are least able to be used together (e.g. a child with a left sixth nerve palsy will turn the head to the left to avoid left gaze, so as to enjoy good binocular single vision in the primary position and right gaze). Abnormal head postures may consist of three components: there may

Correspondence to: Mr J. P. Lee, FRCOphth, Strabismus and Paediatric Service, Moorfields Eye Hospital, City Road, London EC1V 2PD, UK. be a face turn to the left or right, head tilt to left or right, and chin elevation or depression. All three components may be present in certain cases, particularly when the deviation is due to a cyclovertical muscle paresis, and the child should be assessed with and without head position to discover what contribution, if any, this makes to binocular function. Other, rarer ocular causes of abnormal head posture include ptosis, both unilateral and bilateral, congenital motor nystagmus with a null zone, and homonymous hemianopia. Non-ocular causes include unilateral deafness, and spinal muscular problems such as kyphoscoliosis and neck dystonia.

The cover uncover test should be done in all patients to a suitable accommodative target; the demonstration of a manifest ocular deviation, while not excluding binocularity, as this may well exist in an anomalous form, will certainly prove that normal bifoveal fixation is not present. In the absence of a manifest deviation, the cover test and the associated alternate cover test will give clear information as to the presence of binocular function because as the eyes are observed following the removal of the cover it can be seen whether a recovering fusional movement is made. If such a movement is made, the alacrity and efficiency with which it is made are useful and direct clues as to the quality of the underlying binocular function and motor fusion.

By the same argument the assessment of binocular convergence to an interesting toy moving in towards the face will show whether or not the two eyes converge symmetrically to maintain bifoveal fixation on the object of regard. It is possible to have reflex convergence of both eyes without binocular function, but usually it will be clear that the patient is following the target with only one eye and the other is not being used. If there is any doubt a cover test during the convergence movement will make matters clearer.

Finally, in the small child the demonstration that the child overcomes a base-out prism (conventionally $15-20$ prism dioptres) and realigns the visual axes is 
strong corroborative evidence for the presence of binocularfunction. As the prism is removed the eyes are seen to diverge in order to regain binocular fixation.

\section{DEMONSTRATION OF SENSORY AND MOTOR FUSION}

In the older child one can also assess the maintenance of single vision on binocular convergence and in addition the synoptophore may be used to assess the presence of fusional ability and is of particular value where a manifest deviation is present. Appropriate targets with details may be used to exclude suppression and the patient may then have the full range of binocular fusional vergence fully assessed. Children usually enjoy synoptophore testing and once they are capable of giving adequate responses they are usually accurate in their observations.

Fusion range can be tested in free space by observing motor fusion to base-in and base-out variable prisms by using a prism bar or a rotary prism of the Risley variety. Again the demonstration of smooth convergence or divergence without awareness of diplopia and without the development of a manifest deviation is the key observation. Most healthy children will show a convergence range of around 40-50 prism dioptres and a range of divergence of between 10 and 15 prism dioptres.

Sensory tests for fusion include Bagolini lenses in which the patient views a fixation light through lenses with striations at 45 and 135 degrees respectively. The patient fixates a light and gives an appropriate response. If the eyes are straight and there is normal retinal correspondence the patient should see one light and symmetrical streaks of light forming a cross. If there is a manifest deviation but still anomalous correspondence with binocular function the patient will still see a cross. In the presence of diplopia and normal correspondence two lights and two lines are seen separated by an amount appropriate to the size of the manifest deviation.

The Worth Four Dot Test involves viewing four lights either at distance or near, of which one is red, two are green and one is white. If normal fusion is present the patient when viewing the picture through red and green glasses will report the presence of one red light, two green lights and one light which can vary between red and green depending on ocular dominance and retinal rivalry. In the event of the patient suppressing one or other eye they will see either two red lights or three green lights. Diplopic patients will report three green and two red lights, the separation being determined by the angle and direction of squint. The test is easily done and a simple response can be obtained from most children.

\section{DEMONSTRATION OF STEREOPSIS}

Stereopsis is produced by the stimulation of horizontally disparate retinal areas which fall within Panum's fusional areas. Should this sensation of horizontal disparity occur the image will be perceived as one percept with the illusion of depth. The wider the inter-pupillary distance, the greater (in theory) the discrimination achievable.

Stereoacuity is the measure of the minimum perceivable horizontal disparity expressed in seconds of arc. The lower limit of this in adults is around 40 seconds of arc, but it is probable that many normal adult subjects have better stereoacuity than this when tested with appropriate specialised apparatus. Stereopsis can be demonstrated from a few months of age but may continue to develop and increase in its accuracy until about 8 or 9 years old. Tests may be qualitative or quantitative and are based on a variety of optical and other principles.

\section{Qualitative Tests}

In the Lang Two Pencil Test the child is asked to place a pencil on top of one being held vertically by the examiner. It is conventional to do the test with either eye in turn and then with both eyes together, the aim being to assess the accuracy or otherwise of the positioning shown by the subject. Although the test is easy to do, and is certainly a game enjoyed by children, it will detect only very gross deficiencies of stereoacuity and is in no sense an accurate screening test.

The Synoptophore can again be useful in patients who have a manifest deviation. It can be used to assess the potential for binocular single vision or the strength of anomalous retinal correspondence. Slides are usually used which are labelled as being gross or detailed, but there are other slides (Braddick) which are capable of quantifying stereopsis between 90 and 720 seconds of arc. These are based on the random dot principle and can be used in older patients. The synoptophore is enjoyable for children but may give artefactual information of binocular function demonstrable with the synoptophore which is not in practice achievable.

\section{Quantitative Tests}

It is our strong clinical impression that the $T N O$ Stereo Test is a good test for confirming high-grade binocular single vision and stereopsis. It consists of computer-generated red/green random dots viewed at $40 \mathrm{~cm}$ using appropriate complementary red and green glasses. It includes non-quantitative screening plates consisting of pictures of a butterfly, boxes and symbols, a suppression test and a series of quantitative stereoacuity plates measuring between 480 and 15 seconds of arc. The advantage is there are no monocular clues. The disadvantages are that it 
requires red/green glasses to be worn, which some children do not like, and subjects with significant latent deviations or intermittent tropias may perform poorly due to dissociation in the absence of contour clues and have their stereoacuity thereby underestimated. The test seems robust and stands up well to the wear and tear of a busy orthoptic clinic. It is widely regarded as being one of the better clinical practical tests for the demonstration of bifoveal binocular vision.

The Titmus Stereo Test (also known as the Wirt Stereo Test) is a vectograph test in which two targets are polarised at 90 degrees relative to one another and viewed through polaroid filters which give a depth perception illusion. The largest disparity (3000 seconds) is given by the picture of a fly, which takes up half the test and when seen through polaroid spectacles can be so dramatic as to cause small and susceptible children to burst into tears. This positive response will confirm the presence of stereopsis but means it is difficult to continue with the test. There are then three pictures of animals which contain many contour clues and which range in stereo disparity from 400 to 100 seconds, and a series of circles which range in stereo disparity from 800 to 40 seconds of arc. The test is fairly robust but polaroid spectacles have to be worn. These are somewhat flimsy and are easily broken by the more active child. The advantage of the test is ease of response from relatively young children; the disadvantages are that it requires spectacles and that it is not difficult to pick out uniocular clues, thereby leading to inappropriate overestimation of stereoacuity.

The vectograph principle has also been utilised in the testing of distance stereopsis using instruments such as the American optical projection chart. We have limited experience of these devices and they certainly seem to offer some help in the estimation of stereoacuity at distance. The machine that we have used had two stereoacuity slides: one with different geometrical shapes and one with circles polarised in order to stand out from the background. Little has been written on the relationship of stereoacuity to the management of strabismus, in particular that of intermittent exotropia, but systematic studies will doubtless produce more information on this matter.

Another considerable value of a distance vectographic test is that it makes a very good test for simulated visual loss, as patients do not realise that if they read along a whole line of letters some of which are polarised in different phase, they are in fact seeing them with the two eyes at the same time.

The Randot Stereo Test is a polaroid test similar to the Titmus Stereo Test but utilising a random-dot type of target instead of contour targets. It is polarised in the same way and viewed with the same kind of glasses. This test appears to be better than the Titmus test in terms of giving fewer monocular clues, but does not appear to be as good as the TNO test, except in so far as the red/green glasses of the TNO test tend to produce more disassociation in patients with a poorly controlled phoria and therefore lead to poorer results. Another version of the test consists of a random-dot letter 'E'. It is performed at $50 \mathrm{~cm}$ and the child is required to identify which of the two plates contains the E. This test has been used for screening large rural populations for the absence of stereopsis, allowing those who fail to be examined in more detail for strabismus and amblyopia.

The Frisby Stereo Test is a stereo test based on actual depth of target. It consists of three Perspex sheets of different thicknesses and the patient is asked to identify which figure, of four on each plate, is the one that is either closer or further away, the fixation target having been printed on one or the other side of the Perspex sheet. The disparities are between 600 and 15 seconds of arc and it is easy to use in relatively small children who will either look towards the disparate target or may well reach out to grasp it. As glasses are not required it is an easy test to do in a relatively small child. Its disadvantages are that if there is any movement of the plates, or indeed of the patient's head, then it is quite easy to pick out the disparity even monocularly. Nevertheless, it is a relatively easy test to administer.

There are now two different Lang Stereo Tests, both of which are based on the principle of the 'three-dimensional postcard'. These are stout plastic cards with a ribbed surface, one set of images being printed on one slope and another on the opposite slope. If they are then viewed at an appropriate working distance one eye will tend to see the lefthand side of the picture the other eye the right. If a random-dot image is printed onto the postcard then the patient will see something standing out from the background. On card 1 all the targets have stereo disparity and consist of a cat, a car and a star. On the second test there is a crescent moon, a car and an elephant, but in addition there is one target which does not have stereo disparity which the child can identify. This is in order to encourage the child, who will pick at least one of the four targets and thereby does not feel frustrated or disappointed by his or her failure. It is fairly gross test and detects stereo disparities between 1200 and 200 seconds of arc. The test is easy to do and does not require any glasses and its inventor claims that small children will respond to it quite well. The disadvantages are that it is difficult to be sure what the child is grasping or reaching for. It is hard to hold the card parallel to the face without head movement, and unless a verbal response is obtained it is difficult to be sure whether anything has been seen. 
The Awaya Stereo Test appears to be no longer widely available and yet is valuable. It is based on red/green dissociation, comes with its own set of glasses and measures stereoacuities from 4120 down to 40 seconds of arc. It appears to lack monocular clues and perhaps should be more widely known and distributed.

\section{CONCLUSION}

In the first instance, a carefully taken history will give many clues to the presence or absence of binocular vision. By careful clinical examination using appropriate tests, it is relatively easy to confirm the presence of binocularity in subjects whose eyes appear straight on cover testing.

In the presence of a manifest deviation, assessment can establish the state of retinal correspondence, the presence of suppression, or the potential for binocular single vision, and is an essential prerequisite to determining the management plan.

Any well-equipped paediatric ophthalmology or orthoptic department should possess a number of different stereo tests, as the perfect stereo test has yet to be invented. Doubtless, in the future, developments in computer-generated stereograms on VDU monitors will lead to more efficient methods of testing for this important visual function. 